\title{
New Approach to Rayleigh Wave Propagation in the Elastic Halfspace — Viscoelastic Layer Interface
}

\author{
M. PASTERnAK* \\ Institute of Radioelectronics, Military University of Technology \\ S. Kaliskiego 2, 00-490 Warsaw, Poland
}

The Rayleigh wave propagation problem in the elastic halfspace - viscoelastic layer interface was analysed in the paper. The problem was formulated in the Fourier-Laplace space using the Biot viscoelastic solid model. The characteristic equation has taken the Rayleigh equation form with correction term describing viscoelastic layer properties influence on the wave velocity. The approach presented here seems to be useful for surface acoustic waves gas sensors modelling because many chemisensitive coatings applied to the sensors exhibit viscoelastic properties.

PACS numbers: 43.35.Pt, 68.35.Iv, 43.20.Mv, 46.35.+z

\section{Introduction}

There are several papers describing the different approaches to solution of the Rayleigh wave propagation problem in the elastic halfspace - layer interface (e.g. [1-6]). The main reason of the interest is that such descriptions are important from different technologies, and especially from surface acoustic wave (SAW) sensors technology point of view. Unfortunately, the papers usually ignore the viscoelastic properties of the layer or take it into consideration by introducing to the equations complex propagation constant. The approaches, although formally right, usually do not allow to predict the Rayleigh wave velocity change under influence of the viscoelastic layer properties variation, during particle sorption, for instance. It is well known that the existence and value of the influence decides on the most important SAW sensors properties like sensitivity or minimal detectable concentration of sensing particles. Actually, the most of coatings useful to the SAW sensors constructing are polymers. Such materials exhibit strong both geometrical and material properties variations during specific particles sorption and solvatation. Although the changes of the properties may be calculated but the polymer layer influence on the Rayleigh wave velocity was still not described in

*e-mail: mpasternak@wat.edu.pl 
the satisfactory way. The approach presented here seems to fill this gap. The properties of the layer were described in the paper by time dependent Lamé constants using the Biot viscoelastic solid model with Maxwellian relaxation time.

\section{Problem formulation}

Let us consider the viscoelastic layer at the elastic halfspace with thickness $h$ and introduce the Cartesian coordinates, the $x_{3}$ axis is directed into the depth of the substrate (Fig. 1).

The displacements in the substrate and the layer was described using well known potential approach [1]:

$$
\boldsymbol{u}=\operatorname{grad} \phi+\operatorname{rot} \boldsymbol{\psi},
$$

where $\phi$ and $\boldsymbol{\psi}$ are scalar and vector potentials, respectively. For unambiguity the condition $\operatorname{div} \boldsymbol{\psi}=0$ must be additionally satisfied. It is valid for the Rayleigh wave when $\frac{\partial}{\partial x_{2}} \equiv 0$.

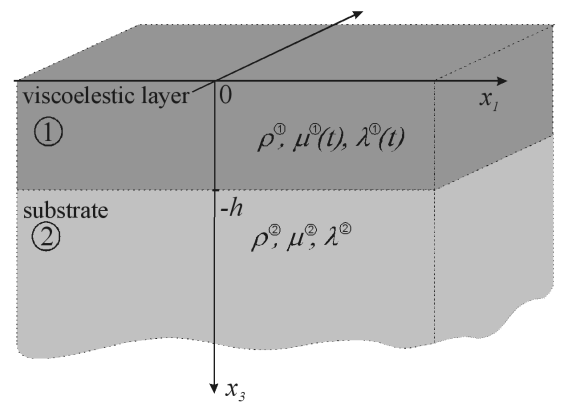

Fig. 1. System under consideration. Values $\rho, \mu$ and $\lambda$ described density and the Lamé constants for the layer (1) and substrate (2), respectively.

The homogeneous wave equations for the potentials in the layer take the form

$$
\begin{aligned}
& \rho \frac{{ }^{(1)}}{\partial t^{2} \phi^{(1)}}-\left(\lambda^{(\Phi}(t)+2 \mu^{(1)}(t)\right) * \nabla^{2} \phi^{(1)}=0, \\
& \rho \frac{{ }^{(1)}}{\partial t^{2}}-\mu^{(1)}(t) * \nabla^{2} \boldsymbol{\psi}^{(1)}=0,
\end{aligned}
$$

where $*$ means the convolution operation and

$$
\begin{aligned}
& \lambda^{\oplus}(t)=\lambda_{0} \delta(t-\tau)-\frac{\lambda^{\Phi}}{\tau} \exp \left(-\frac{t}{\tau}\right), \\
& \mu^{(}(t)=\mu_{0} \delta(t-\tau)-\frac{\mu^{\circledR}}{\tau} \exp \left(-\frac{t}{\tau}\right) .
\end{aligned}
$$

The $\mu_{0}$ and $\lambda_{0}$ are instantaneous Lamé constants for the layer, $\tau$ — viscoelastic solid Maxwellian relaxation time. It is assumed that the relaxation times 
for both Lamé constants are the same. The validity of such assumption was empirically proved for a great deal of amorphic polymer layers.

In the elastic substrate case the wave equations take the following form:

$$
\begin{aligned}
& \rho \frac{\partial^{2} \phi^{(2)}}{\partial t^{2}}-\left(\lambda^{(2)}+2 \mu^{(2)}\right) \nabla^{2} \phi^{(2)}=0, \\
& \rho^{(2)} \frac{\partial^{2} \boldsymbol{\psi}^{(2)}}{\partial t^{2}}-\mu^{(2)} \nabla^{2} \psi^{(2)}=0 .
\end{aligned}
$$

After the Laplace and Fourier transformations the potentials for the layer take the form

$$
\begin{aligned}
& \hat{\bar{\phi}}^{(1)}\left(x_{3} ; \alpha ; s\right)=A^{(\mathbb{1}} \exp \left(-\bar{\gamma}_{1}^{\circledR} x_{3}\right)+B^{(\mathbb{1}} \exp \left(-\bar{\gamma}_{1}^{(}\left(2 h-x_{3}\right)\right), \\
& \hat{\bar{\psi}}_{2}^{(}\left(x_{3} ; \alpha ; s\right)=C_{2}^{(\mathbb{1}} \exp \left(-\bar{\gamma}_{2}^{(1} x_{3}\right)+D_{2}^{(\mathbb{1}} \exp \left(-\bar{\gamma}_{2}^{(1}\left(2 h-x_{3}\right)\right),
\end{aligned}
$$

where $\bar{\gamma}_{1}^{\circledR}=\sqrt{\frac{\left.s^{2} \rho^{(}\right)}{\lambda^{\circledR}(s)+2 \mu^{\Phi}(s)}+\alpha^{2}}, \bar{\gamma}_{2}^{\circledR}=\sqrt{\frac{s^{2} \rho^{(}}{\mu^{\circledR}(s)+\alpha^{2}}+\alpha^{2}}, \alpha$ and $s$ are the Fourier and Laplace transformations parameters, respectively.

For the substrate

$$
\begin{aligned}
& \hat{\bar{\phi}}^{(2)}\left(x_{3} ; \alpha ; s\right)=A^{(2)} \exp \left(-\gamma_{1}^{(\mathbb{1}} x_{3}\right), \\
& \hat{\bar{\psi}}_{2}^{(2)}\left(x_{3} ; \alpha ; s\right)=C_{2}^{(2)} \exp \left(-\gamma_{2}^{(\mathbb{1}} x_{3}\right),
\end{aligned}
$$

where $\bar{\gamma}_{1}^{(2)}=\sqrt{\frac{s^{2} \rho^{(2)}}{\lambda^{(2}+2 \mu}+\alpha^{2}}$, and $\bar{\gamma}_{2}^{(2)}=\sqrt{\frac{s^{2} \rho^{(2)}}{\mu^{(2)}}+\alpha^{2}}$.

Inserting Eqs. $(8) \div(11)$ into the $(1)$ the displacements in the layer obtain the form

$$
\begin{aligned}
& \hat{\bar{\mu}}_{1}^{(1)}=\mathrm{i} \alpha\left(\bar{A}^{(} \exp \left(-\bar{\gamma}_{1}^{\circledR} x_{3}\right)+\bar{B}^{(} \exp \left(-\bar{\gamma}_{1}^{(}\left(2 h-x_{3}\right)\right)\right) \\
& +\bar{\gamma}_{2}^{(1)} \bar{C}_{2}^{(} \exp \left(-\bar{\gamma}_{2}^{\circledR} x_{3}\right)-\bar{\gamma}_{2}^{(1} \bar{D}_{2}^{(1)} \exp \left(-\bar{\gamma}_{2}^{(1}\left(2 h-x_{3}\right)\right), \\
& \hat{\bar{\mu}}_{3}^{(1)}=-\bar{\gamma}_{1}^{(\mathbb{1}} \bar{A}^{(\mathbb{1})} \exp \left(-\bar{\gamma}_{1}^{(1)} x_{3}\right)+\bar{\gamma}_{1}^{(\mathbb{1}} \bar{B}^{(\mathbb{1}} \exp \left(-\bar{\gamma}_{1}^{(1}\left(2 h-x_{3}\right)\right) \\
& +\mathrm{i} \alpha\left(\bar{C}_{2}^{(1)} \exp \left(-\bar{\gamma}_{2}^{(1} x_{3}\right)+\bar{D}_{2}^{(\mathbb{1}} \exp \left(-\bar{\gamma}_{2}^{(1}\left(2 h-x_{3}\right)\right)\right)
\end{aligned}
$$

and in the substrate

$$
\begin{aligned}
& \hat{\bar{\mu}}_{1}^{(2)}=\mathrm{i} \alpha A^{(2)} \exp \left(-\gamma_{1}^{(2)} x_{3}\right)+\bar{\gamma}_{2}^{(2} \bar{C}_{2}^{(2)} \exp \left(-\gamma_{2}^{(2)} x_{3}\right), \\
& \hat{\bar{\mu}}_{3}^{(2)}=-\bar{\gamma}_{1}^{(2} \bar{A}^{(2)} \exp \left(-\gamma_{1}^{(2} x_{3}\right)+\mathrm{i} \alpha C_{2}^{(2)} \exp \left(-\gamma_{2}{ }^{(2)} x_{3}\right) .
\end{aligned}
$$

Using the relation for deformation tensor $\varepsilon_{i k}^{(12)}=\frac{1}{2}\left(u_{i, k}^{(12)}+u_{k, i}^{(\mathbb{1} 2)}\right)$ and the Hooke law, one can obtain the stresses according to the relation

$$
\sigma_{i j}^{(12)}=\lambda^{\text {(1) }} \odot \operatorname{tr} \varepsilon \delta_{i j}+2 u^{\text {(1) }} \odot \varepsilon_{i j},
$$

where sign $\odot$ means the convolution in the viscoelastic and an ordinary multiplication for the elastic case. Inserting $(12) \div(15)$ into the deformation formula and next into the (16) one can obtain the following formulae for the stresses 


$$
\begin{aligned}
& \hat{\bar{\sigma}}_{13}^{\oplus}=\hat{\bar{\sigma}}_{31}^{\oplus}=2 \mathrm{i} \alpha \bar{\gamma}_{1}^{\oplus} \bar{\mu}^{\oplus}\left[-\bar{A}^{\oplus} \exp \left(-\bar{\gamma}_{1}^{\oplus} x_{3}\right)+\bar{B}^{\oplus} \exp \left(-\bar{\gamma}_{1}^{\oplus}\left(2 h-x_{3}\right)\right)\right] \\
& -\bar{\mu}^{\oplus}\left\{\left[\alpha^{2}-\left(\bar{\gamma}_{2}^{\circledR}\right)^{2}\right]\left[\bar{C}_{2}^{\circledR} \exp \left(-\bar{\gamma}_{2}^{\circledR} x_{3}\right)+\bar{D}_{2}^{\Phi} \exp \left(-\bar{\gamma}_{2}^{\oplus}\left(2 h-x_{3}\right)\right)\right]\right\}, \\
& \hat{\bar{\sigma}}_{33}^{(}=-\bar{\lambda}^{\circledR} \alpha^{2}\left[\bar{A}^{\oplus} \exp \left(-\gamma_{1}^{\oplus} x_{3}\right)+\bar{B}^{\oplus} \exp \left(-\bar{\gamma}_{1}^{\oplus}\left(2 h-x_{3}\right)\right)\right] \\
& +\left(\bar{\lambda}^{\oplus}+2 \bar{\mu}^{\oplus}\right)\left(\bar{\gamma}_{1}^{\oplus}\right)^{2}\left[\bar{A}^{\oplus} \exp \left(-\gamma_{1}^{\circledR} x_{3}\right)+\bar{B}^{\oplus} \exp \left(-\bar{\gamma}_{1}^{\oplus}\left(2 h-x_{3}\right)\right)\right] \\
& +2 \mathrm{i} \alpha \bar{\gamma}_{2}^{\oplus} \bar{\mu}^{\oplus}\left[-\bar{C}_{2}^{\oplus} \exp \left(-\bar{\gamma}_{2}^{\oplus} x_{3}\right)+\bar{D}_{2}^{\oplus} \exp \left(-\bar{\gamma}_{2}^{\oplus}\left(2 h-x_{3}\right)\right)\right],
\end{aligned}
$$

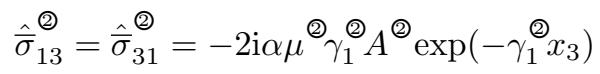

$$
\begin{aligned}
& -\mu^{(}\left[\alpha^{2}+\left(\gamma_{2}^{(}\right)^{2}\right] C_{2}^{(} \exp \left(-\gamma_{2}^{(} x_{3}\right) \text {, } \\
& \hat{\bar{\sigma}}_{33}^{\circledR}=\left[-\lambda^{\oplus} \alpha^{2}+\left(\lambda^{\oplus}+2 \mu^{\Theta}\right)\left(\gamma_{1}^{\Theta}\right)^{2}\right] A^{\oplus} \exp \left(-\gamma_{1}^{\circledR} x_{3}\right) \\
& -2 \mathrm{i} \alpha \mu^{(}\left(\gamma_{2}^{\circledR} C_{2}^{\circledR} \exp \left(-\gamma_{2}^{\circledR} x_{3}\right)\right) \text {. }
\end{aligned}
$$

The boundary conditions assume vanishing of the stresses at the top of the layer

$$
\sigma_{13}^{(}=0, \quad \sigma_{33}^{\circledR}=0 \quad \text { for } \quad x_{3}=0
$$

and equality of respective displacements and stresses at the interface

$$
u_{1}^{(}=u_{1}^{(}, \quad u_{3}^{(}=u_{3}^{(}, \quad \sigma_{13}^{(}=\sigma_{13}^{\circledR}, \quad \sigma_{33}^{(}=\sigma_{33}^{(} \quad \text { for } \quad x_{3}=-h .
$$

Inserting the displacements and stresses into the boundary conditions (21) and (22) one can obtain the homogeneous equation system. In the matrix notation it has the form

$$
[\boldsymbol{M}]\left[A^{\oplus} A^{\oplus} B^{\oplus} C_{2}^{\oplus} C_{2}^{\oplus} D_{2}^{\Phi}\right]^{\mathrm{T}}=0 .
$$

The system (23) has nontrivial solution when the characteristic equation $\operatorname{det}[\boldsymbol{M}]=0$ is satisfied. Unfortunately, the determinant has long and complicated form.

In order to simplify it was developed into multi Taylor series around $\exp \left(-\bar{\gamma}_{1}^{\oplus} h\right)=0, \exp \left(-\bar{\gamma}_{2}^{\circledR} h\right)=0, \exp \left(-\bar{\gamma}_{1}^{\circledR} h\right)=0, \exp \left(-\bar{\gamma}_{2}^{\Theta} h\right)=0$, and limited to the two terms. This means that there are possible two bulk waves reflections from the top of the layer only. It is physically justified for viscoelastic medium with relaxation time long enough.

After such simplification one term of the determinant should have well known Rayleigh equation form for free elastic surface. Indeed, after factorisation of simplified determinant one of factors has the Rayleigh equation form. The rest of factors describe the modification of the Rayleigh equation - correction term with viscoelastic origin. This term vanishes towards the relaxation time. 
In order to separate the correction term the following substitution has been applied:

$\alpha=s x$

and

$$
\begin{aligned}
& \bar{\gamma}_{1}^{(1)}=s f_{1}(s) \sqrt{v_{11}^{2}+x^{2}}, \quad \bar{\gamma}_{2}^{(1)}=s f_{2}(s) \sqrt{v_{21}^{2}+x^{2}}, \\
& \bar{\gamma}_{1}^{(\mathcal{Q}}=s \sqrt{v_{12}^{2}+x^{2}}, \quad \bar{\gamma}_{2}^{()}=s \sqrt{v_{22}^{2}+x^{2}},
\end{aligned}
$$

where $v_{11}, v_{21}, v_{12}, v_{22}$ mean reverses of velocities of longitudinal and transversal waves in the layer and longitudinal and transversal waves in the substrate, respectively, $x$ is interpreted as a reverse of the Rayleigh wave at the free surface of the substrate. The functions $f_{1}(s)$ and $f_{2}(s)$ come from the Laplace transformations of the Maxwellian relaxation times and take the form

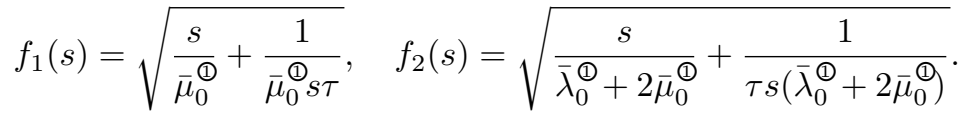

It is assumed that $f_{1}(s) / f_{2}(s)$ depends on the Poisson ratio only and not relaxes. The above assumptions and substitutions allow to eliminate transformation parameter $s$ from the equation.

That way the independent of $s$ dispersion equation was obtained. It has the form of sum of the Rayleigh equation and correction term

$$
\boldsymbol{R}+\boldsymbol{R}_{\text {corr }}=0 \text {. }
$$

The correction term has rather complicated form, but it is fit for numerical calculation. It consists on certain combination of algebraic equation containing the material constants and thickness of the layer. The numerical calculations for real substrates and layer materials showed that the correction term is a negative number with order of magnitude lower value than the Rayleigh wave. In such depiction the Rayleigh wave velocity change is caused by correction term variation.

\section{Conclusions}

The results of calculation allow to conclude that the Rayleigh wave velocity along the elastic substrate — viscoelastic layer interface depends mainly on correction component value of the characteristic equation. It depends on transversal and longitudinal bulk waves velocities in the layer and the layer thickness. According to the conclusion the operation principle of the Rayleigh wave gas sensors with polymeric layer can be interpreted in alternative way. The change in SAW velocity in the sensor (determining its sensitivity) may be explained not as a layer mass change but as a result of its solvation — bulk waves velocities decrease.

\section{References}

[1] A. Auld, Acoustic Fields and Waves in Solids, Wiley Interscience, New York 1973.

[2] S.J. Martin, G.C. Frye, S.D. Senturia, Anal. Chem. 66, 2201 (1994). 
[3] A. Snakowska, Mol. Quant. Acoust. 28, 241 (2007).

[4] E. Nazarova, A. Tcheryatian, Mol. Quant. Acoust. 28, 259 (2007).

[5] V. Balakshy, A. Vostrikova, Mol. Quant. Acoust. 28, 265 (2007).

[6] G. McHale, R. Lücklum, M.I. Newton, J.A. Cowen, J. Appl. Phys. 88, 7304 (2000). 